\title{
ASPECTOS FLORÍSTICOS E ECOLÓGICOS DO CAMPUS REGIONAL DA UNIVERSIDADE FEDERAL DE MINAS GERAIS EM MONTES CLAROS - MG
}

\author{
FLORISTIC AND ECOLOGICAL ASPECTS OF THE REGIONAL CAMPUS OF THE \\ FEDERAL UNIVERSITY OF MINAS GERAIS IN MONTES CLAROS - MG
}

Jonathan Ezequiel da Silveira ${ }^{1}$, Carlos Emílio de Sant’Ana Pinter Pastorello¹, Rúbia Santos Fonseca²

\section{RESUMO}

Objetivou-se caracterizar a comunidade arbórea das vias e jardins do Campus Regional da Universidade Federal de Minas Gerais em Montes Claros, norte de Minas Gerais, região marcada pelo ecótono Caatinga-Cerrado-Mata Atlântica. Buscou-se descrever sua riqueza, origem e grau de conservação das espécies e o potencial de interação com a fauna. Foram coletados materiais botânicos das espécies arbóreas lenhosas e não lenhosas arborescentes presentes nas vias e jardins do campus, sendo posteriormente, herborizados e identificados. A caracterização das síndromes de polinização e dispersão foi realizada por meio de atributos morfológicos (flores, frutos e sementes). Foram inventariadas 122 espécies, pertencentes a 33 famílias botânicas. A maior parte das espécies eram nativas do Brasil $(57,4 \%)$, destas, $87 \%$ ocorrem naturalmente na região norte mineira. A presença de exemplares de espécies nativas e exóticas ameaçadas de extinção, além de outras protegidas pela legislação, evidenciam o papel conservacionista proposto pela silvicultura urbana. Melitofilia e zoocoria foram, respectivamente, as síndromes de polinização e dispersão mais representativas, demonstrando o potencial de interação da flora do campus com a fauna local, destacando-se as abelhas.

Palavras-chave: Áreas verdes; Conservação; Dispersão; Polinização; Silvicultura Urbana.

\begin{abstract}
This study aimed to know the arboreal community of the roads and gardens of the Regional Campus of the Federal University of Minas Gerais in Montes Claros, north of Minas Gerais, a region marked by the Caatinga-Cerrado-Atlantic forest ecotone. We seek to describe the richness, origin and degree of conservation of the species and the potential for interaction with the fauna. Botanical materials of trees and non-woody arborescent species present in the roads and gardens of the campus were collected, herborized and identified. The characterization of pollination and dispersion syndromes was carried out through morphological attributes (flower, fruits and seeds). A total of 122 species were inventoried, belonging to 33 botanical families. Most of the species were native to Brazil (57.4\%), of these $87 \%$ occur naturally in the northern region of Minas Gerais. The presence of native and exotic species threatened, in addition to others protected by legislation, highlights the conservationist role proposed by urban forestry. Melittophily and zoochory were the most representative pollination and dispersion syndromes, respectively, demonstrating the potential for interaction between the flora of the campus and the local fauna, especially bees.
\end{abstract}

Keywords: Conservation; Dispersal; Green areas; Pollination; Urban Forestry.

Recebido em 26.05.2020 e aceito em 07.07.2020

1. Acadêmicos do curso de Engenharia Florestal da UFMG. Montes Claros/MG. Emails: jonathan.evangel@hotmail.com / carlospastorello@hotmail.com

2. Bióloga. Doutora em Botânica. Professora adjunta de Sistemática Vegetal e Dendrologia da UFMG / Curadora do Herbário Norte Mineiro (MCCA). Montes Claros/MG. Email: rubiafonseca@hotmail.com 


\section{INTRODUÇÃO}

A arborização urbana devidamente planejada é uma importante ferramenta para a atenuação e solução de problemas ambientais urbanos (PATRÍCIO, 2017). Ao prestar diversos serviços ecossistêmicos, as áreas verdes urbanas reduzem o impacto de eventos climáticos extremos (GILL et al., 2007), trazem benefícios para saúde humana e promovem a valorização estética e imobiliária de ruas e bairros (PATRícIO, 2017). Em relação ao aspecto ecológico, essas áreas além de fornecerem alimentação, habitação e locais de nidificação para a fauna local (CASTRO; MORO; ROCHA, 2011), possibilitam a conservação in situ e ex situ da flora, contribuindo para a manutenção de espécies ameaçadas de extinção (CUPERTINO; EISENLOHR, 2013).

Os campi universitários estão entre os exemplos de áreas verdes presentes em algumas cidades brasileiras, como Montes Claros, no norte de Minas Gerais. Considerado polo regional e centro universitário, o município possui população estimada em mais de 400 mil habitantes (PMMC, 2020), abrigando diversas instituições de ensino superior públicas e privadas, dentre as quais está o Campus Regional da Universidade Federal de Minas Gerais (UFMG).

O norte de Minas Gerais é marcado pelo encontro de três domínios morfoclimáticos, Caatinga, Cerrado e Mata Atlântica, possibilitando a ocorrência de rica biodiversidade, com muitas espécies endêmicas e ameaçadas de extinção (ARRUDA et al., 2013). Apesar disso, as espécies autóctones são comumente minoria na flora urbana desta região (VELOSO et al., 2015; FIRMO et al., 2019).

A despeito de ser uma importante área verde de Montes Claros, a arborização do Campus Regional da UFMG é desconhecida. Assim, o objetivo deste trabalho foi responder às seguintes perguntas: Qual a composição florística da comunidade arbórea do campus? Existem espécies ameaçadas de extinção e/ou protegidas pela legislação? Qual a proporção de espécies nativas e exóticas? Esta comunidade reflete o caráter ecotonal da região norte de Minas Gerais? Quais são as síndromes de polinização e dispersão desta comunidade? Qual o potencial de interação com a fauna local?

\section{MATERIAL E MÉTODOS}

\section{Área de estudo}

O Campus Regional da UFMG está situado no município de Montes Claros (Figura 1), região norte de Minas Gerais. A cidade é caracterizada por apresentar altitude média de $638 \mathrm{~m}$, clima tropical, classificado como Aw segundo Köppen-Geiger, com altas temperaturas ao longo do ano e chuvas concentradas no verão (FIRMO et al., 2019; PMMC, 2020). 
O Campus Regional foi criado em 1964 como Colégio Agrícola Antônio Versiani Athayde e incorporado à UFMG em 1968. A unidade acadêmica apresenta jardins e vias arborizadas que associados a existência, em sua propriedade, de fragmentos de cerrado sensu stricto, floresta estacional decidual ("matas secas"), matas ciliares e elementos florísticos da Caatinga, a torna uma importante área verde da cidade, além de corroborar o caráter ecotonal observado na região norte mineira.

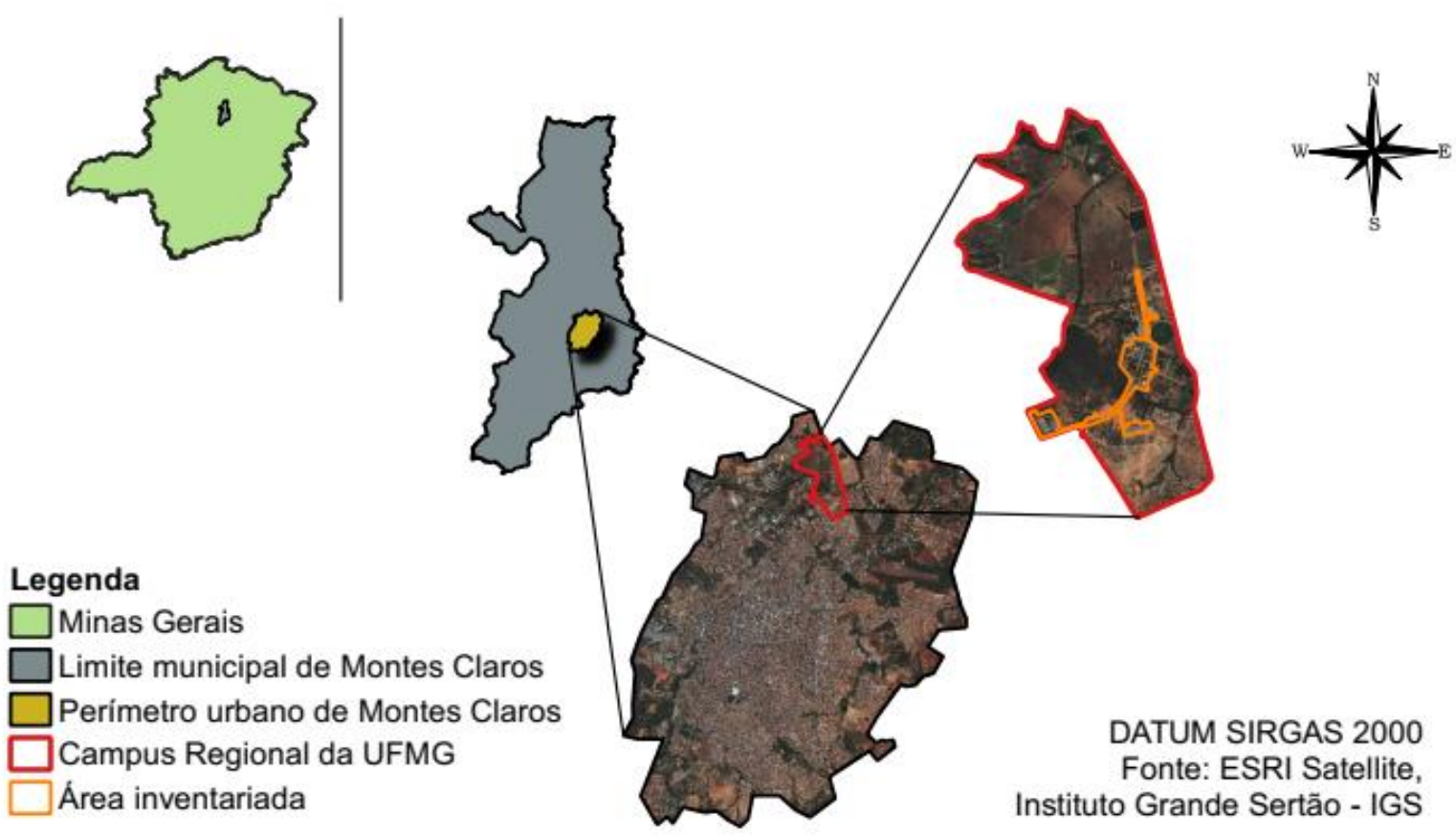

Figura 1. Localização do Campus Regional da Universidade Federal de Minas Gerais no município de Montes Claros, Minas Gerais e detalhes das vias e jardins

Figure 1. Location of the Regional Campus of the Federal University of Minas Gerais in the municipality of Montes Claros, Minas Gerais and details of the roads and gardens

\section{Levantamento florístico e classificação das síndromes de dispersão e polinização}

O levantamento florístico foi realizado entre março de 2017 e julho de 2019, por meio de caminhadas pelas vias e jardins do Campus Regional (Figura 1). Foi coletado material botânico fértil das espécies arbóreas e arborescentes, que foram herborizados, identificados por meio de literaturas especializadas e depositados no Herbário Norte Mineiro (MCCA). A nomenclatura botânica e a origem das espécies foram determinadas segundo a base de dados do Flora do Brasil (2020) e The Plant List (2020).

A classificação das síndromes de polinização baseou-se na morfologia, recursos florais e comportamento da flor (sensu FAEGRI; PIJL, 1976), sendo divididas em: anemofilia, cantarofilia entomofilia, falenofilia, melitofilia, ornitofilia e quiropterofilia. As síndromes de dispersão, baseadas na morfologia dos frutos (sensu VAN DER PIJL, 1982), foram classificadas em: anemocoria, autocoria, barocoria, hidrocoria e zoocoria. 


\section{RESULTADOS E DISCUSSÃO}

Foram catalogadas 120 espécies de angiospermas e duas de gimnospermas, distribuídas em 33 famílias botânicas (Tabela 1). As famílias mais ricas foram Fabaceae (30 espécies), Arecaceae (13), Bignoniaceae (13), Anacardiaceae (11) e Myrtaceae (7), que juntas englobam cerca de $61 \%$ de todas as espécies da arborização do campus. A preferência por estas famílias, com exceção de Anacardiaceae, é reportada em outros campi universitários pelo país (CUPERTINO; EISENLOHR, 2013) e na arborização urbana de Montes Claros (VELOSO et al., 2015; FIRMO et al., 2019). A grande representatividade de Anacardiaceae pode estar relacionada a sua importância estrutural e florística em áreas próximas ao campus (DURÃES et al., 2014) e ao uso recorrente de suas espécies nos quintais do norte de Minas Gerais, especialmente as do gênero Spondias, muito apreciadas regionalmente (DUQUE-BRASIL et al., 2012).

Tabela 1. Famílias, espécies e atributos ecológicos da arborização do Campus Regional da Universidade Federal de Minas Gerais, Montes Claros, MG

Table 1. Families, species and ecological attributes of the afforestation of the Regional Campus of the Federal University of Minas Gerais, Montes Claros, MG

\begin{tabular}{|c|c|c|c|c|c|}
\hline Família & Nome científico & Nome popular & Origem & SP & SD \\
\hline \multirow{11}{*}{ Anacardiaceae } & Anacardium occidentale L. & cajuzeiro & NO & Mel. & Zoo. \\
\hline & Astronium fraxinifolium Schott & gonçalo-alves & NR & Mel. & Anec. \\
\hline & Astronium urundeuva (M. Allemão) Engl. & aroeira-do-sertão & NR & Mel. & Anec. \\
\hline & Mangifera indica L. & mangueira & $\mathrm{E}$ & Ent. & Zoo. \\
\hline & Schinopsis brasiliensis Engl. & pau-preto & NR & Mel. & Anec. \\
\hline & Schinus molle L. & aroeira-salsa & NO & Ent. & Anec. \\
\hline & Schinus terebinthifolius Raddi & aroeira-vermelha & NR & Ent. & Zoo. \\
\hline & Spondias mombin $\mathrm{L}$. & cajazeira & NR & Ent. & Zoo. \\
\hline & Spondias purpurea L. & siriguela & $E$ & Ent. & Zoo. \\
\hline & Spondias tuberosa Arruda & umbuzeiro & NR & Ent. & Zoo. \\
\hline & Spondias dulcis Parkinson & cajá-manga & $E$ & Ent. & Zoo. \\
\hline Apocynaceae & $\begin{array}{c}\text { Aspidosperma tomentosum Mart. } \\
\text { Plumeria rubra L. }\end{array}$ & $\begin{array}{c}\text { guatambu } \\
\text { jasmim-manga }\end{array}$ & $\begin{array}{c}\mathrm{NR} \\
\mathrm{E}\end{array}$ & $\begin{array}{l}\text { Fal. } \\
\text { Fal. }\end{array}$ & $\begin{array}{l}\text { Anec. } \\
\text { Anec. }\end{array}$ \\
\hline \multirow{8}{*}{ Arecaceae } & Acrocomia aculeata (Jacq.) Lodd. ex Mart. & macaúba & NR & Can. & Zoo. \\
\hline & Bismarckia nobilis Hildebr. \& H.Wendl & palmeira-azul & $E$ & Ent. & Zoo. \\
\hline & Butia capitata (Mart.) Becc. & coquinho-azedo & NR & Mel. & Zoo. \\
\hline & Caryota urens L. & $\begin{array}{l}\text { palmeira-rabo- } \\
\text { de-peixe }\end{array}$ & $E$ & Ent. & Zoo. \\
\hline & $\begin{array}{c}\text { Coccothrinax argentea (Lodd. ex Schult. \& } \\
\text { Schult.f.) Sarg. ex Becc. }\end{array}$ & $\begin{array}{l}\text { palmeira- } \\
\text { prateada-de- } \\
\text { leque }\end{array}$ & $E$ & Mel. & Zoo. \\
\hline & Cocos nucifera L. & coqueiro & $E$ & Mel. & hidro. \\
\hline & Dypsis decaryi (Jum.) Beentje \& J.Dransf. & $\begin{array}{l}\text { palmeira- } \\
\text { triângulo }\end{array}$ & $E$ & Ent. & Zoo. \\
\hline & $\begin{array}{c}\text { Dypsis lutescens (H.Wendl.) Beentje \& } \\
\text { J.Dransf. }\end{array}$ & palmeira-areca & $E$ & Mel. & Zoo. \\
\hline
\end{tabular}




\begin{tabular}{|c|c|c|c|c|c|}
\hline & \\
\hline & Livistona chinensis (Jacq.) R.Br. ex Mart. & $\begin{array}{l}\text { palmeira-leque- } \\
\text { da-china }\end{array}$ & $E$ & Ent. & Zoo. \\
\hline & Phoenix roebelenii O'Brien & fênix & $E$ & Ent. & Zoo. \\
\hline & Phoenix dactylifera L. & tamareira & $E$ & Ent. & Zoo. \\
\hline & Roystonea regia (Kunth) O.F.Cook & palmeira-real & $E$ & Mel. & Zoo. \\
\hline & Syagrus romanzoffiana (Cham.) Glassman & jerivá & NR & Mel. & Zoo. \\
\hline Asparagaceae & Yucca gigantea Lem. & iuca & $\mathrm{E}$ & Fal. & Anec. \\
\hline \multirow{13}{*}{ Bignoniaceae } & Cybistax antisyphilitica (Mart.) Mart. & ipê-verde & NR & Mel. & Anec. \\
\hline & $\begin{array}{c}\text { Handroanthus chrysotrichus (Mart. ex A. } \\
\text { DC.) Mattos }\end{array}$ & ipê-amarelo & NR & Mel. & Anec. \\
\hline & $\begin{array}{l}\text { Handroanthus impetiginosus (Mart. ex DC.) } \\
\text { Mattos }\end{array}$ & ipê-roxo & NR & Mel. & Anec. \\
\hline & Handroanthus ochraceus (Cham.) Mattos & ipê-amarelo & NR & Mel. & Anec. \\
\hline & Handroanthus serratifolius (Vahl) S.Grose & ipê-amarelo & NR & Mel. & Anec. \\
\hline & Jacaranda brasiliana (Lam.) Pers. & carobão & NR & Mel. & Anec. \\
\hline & Jacaranda cuspidifolia Mart. & caroba & NR & Mel. & Anec. \\
\hline & Kigelia cf. africana (Lam.) Benth. & árvore-salcicha & $E$ & Mel. & Anec. \\
\hline & Spathodea campanulata P. Beauv. & espatódea & $E$ & Ornito. & Anec. \\
\hline & $\begin{array}{c}\text { Tabebuia aurea (Silva Manso) Benth. \& } \\
\text { Hook.f. ex S.Moore }\end{array}$ & $\begin{array}{l}\text { ipê-amarelo-do- } \\
\text { cerrado }\end{array}$ & NR & Mel. & Anec. \\
\hline & Tabebuia rosea (Bertol.) DC. & ipê-rosa & $E$ & Mel. & Anec. \\
\hline & Tabebuia roseoalba (Ridl.) Sandwith & ipê-branco & NR & Mel. & Anec. \\
\hline & Tecoma stans (L.) Juss. ex Kunth & ipê-mirim & $\mathrm{E}$ & Mel. & Anec. \\
\hline Bixaceae & Bixa orellana L. & urucum & NO & Mel. & Zoo. \\
\hline Calophyllaceae & Calophyllum brasiliense Cambess. & guanandi & NR & Mel. & Zoo. \\
\hline Caryocaraceae & Caryocar brasiliense Cambess. & pequizeiro & NR & Quirop. & Zoo. \\
\hline Casuarinaceae & Casuarina equisetifolia L. & casuarina & $E$ & Anemo. & Anec. \\
\hline Chrysobalanaceae & Licania tomentosa (Benth.) Fritsch & oiti & NO & Mel. & Zoo. \\
\hline \multirow[b]{2}{*}{ Combretaceae } & Terminalia argentea Mart. & capitão-do-mato & NR & Ent. & Anec. \\
\hline & Terminalia mantaly H.Perrier & $\begin{array}{l}\text { amendoeira-de- } \\
\text { madagáscar }\end{array}$ & $E$ & Ent. & Anec. \\
\hline Cupressaceae & Cupressus cf. Iusitanica Mill. & cipreste & $\mathrm{E}$ & Anemo. & Anec. \\
\hline Cycadaceae & Cycas circinalis L. & cica & $E$ & Anemo. & Zoo. \\
\hline Dilleniaceae & Curatella americana L. & lixeira & NR & Mel. & Zoo. \\
\hline \multirow[t]{5}{*}{ Euphorbiaceae } & $\begin{array}{c}\text { Hevea brasiliensis (Willd. ex A.Juss.) } \\
\text { Müll.Arg. }\end{array}$ & seringueira & NO & Mel. & Auto. \\
\hline & Acacia mangium Willd. & acácia-mangium & $E$ & Ent. & Zoo. \\
\hline & Adenanthera pavonina L. & olho-de-pavão & $E$ & Mel. & Auto. \\
\hline & Albizia lebbeck (L.) Benth. & albízia & $E$ & Quirop. & Auto. \\
\hline & Albizia niopoides (Spruce ex Benth.) Burkart & farinha-seca & NR & Mel. & Auto. \\
\hline \multirow[t]{4}{*}{ Fabaceae } & Amburana cearensis (Allemão) A.C. Sm. & $\begin{array}{l}\text { amburana-de- } \\
\text { cheiro }\end{array}$ & NR & Mel. & Anec. \\
\hline & Anadenanthera colubrina (Vell.) Brenan & anjico-vermelho & NR & Mel. & Auto. \\
\hline & Bauhinia variegata $\mathrm{L}$. & pata-de-vaca & $E$ & Quirop. & Auto. \\
\hline & Cassia grandis L. f. & cássia-rosa & $\mathrm{NO}$ & Mel. & Auto. \\
\hline
\end{tabular}




\begin{tabular}{|c|c|c|c|c|}
\hline $\begin{array}{c}\text { Cenostigma pluviosum (DC.) E. Gagnon \& } \\
\text { G.P. Lewis }\end{array}$ & sibipuruna & NR & Mel. & Auto. \\
\hline Copaifera langsdorffii Desf. & pau-d'óleo & NR & Ent. & Zoo. \\
\hline Delonix regia (Bojer ex Hook.) Raf. & flamboyant & $E$ & Mel. & Auto. \\
\hline Dipteryx alata Vogel & baruzeiro & NR & Mel. & Bar. \\
\hline Enterolobium contortisiliquum (Vell.) Morong & tamboril & NR & Mel. & Bar. \\
\hline Gliricidia sepium (Jacq.) Kunth ex Walp. & gliricídia & $E$ & Mel. & Auto. \\
\hline Hymenaea courbaril L. & jatobá & NR & Quirop. & Zoo. \\
\hline Hymenaea stigonocarpa Mart. ex Hayne & $\begin{array}{l}\text { jatobá-do- } \\
\text { cerrado }\end{array}$ & NR & Quirop. & Zoo. \\
\hline Inga edulis Mart. & ingazeiro & NO & Quirop. & Zoo. \\
\hline $\begin{array}{c}\text { Lachesiodendron viridiflorum (Kunth) } \\
\text { P.G.Ribeiro et al. }\end{array}$ & surucucu & NR & Ent. & Auto. \\
\hline Leptolobium dasycarpum Vogel & unha-de-gato & NR & Mel. & Anec. \\
\hline Leucaena leucocephala (Lam.) de Wit & leucena & $E$ & Mel. & Auto. \\
\hline Libidibia ferrea (Mart. ex Tul.) L.P. Queiroz & pau-ferro & NR & Mel. & Auto. \\
\hline Machaerium hirtum (Vell.) Stellfeld & $\begin{array}{l}\text { jacarandá-bico- } \\
\text { de-pato }\end{array}$ & NR & Mel. & Anec. \\
\hline Machaerium opacum Vogel & $\begin{array}{l}\text { jacarandá-do- } \\
\text { cerrado }\end{array}$ & NR & Mel. & Anec. \\
\hline Peltophorum dubium (Spreng.) Taub. & canafístula & NR & Mel. & Auto. \\
\hline Pterogyne nitens Tul. & amendoim-bravo & NR & Mel. & Anec. \\
\hline Senna multijuga (Rich.) H.S.Irwin \& Barneby & pau-cigarra & NR & Mel. & Auto. \\
\hline Senna silvestris (Vell.) H.S.Irwin \& Barneby & aleluia & NR & Mel. & Auto. \\
\hline Tamarindus indica L. & tamarindo & E & Mel. & Zoo. \\
\hline Tipuana tipu (Benth.) Kuntze & tipuana & $E$ & Mel. & Anec. \\
\hline Vatairea macrocarpa (Benth.) Ducke & amargoso & NR & Mel. & Anec. \\
\hline Callicarpa nudiflora Hook. \& Arn. & calicarpa & $\mathrm{E}$ & Mel. & Zoo. \\
\hline Couroupita guianensis Aubl. & $\begin{array}{l}\text { abricó-de- } \\
\text { macaco }\end{array}$ & NO & Mel. & Bar. \\
\hline Lagerstroemia speciosa (L.) Pers. & rosedá-gigante & $\mathrm{E}$ & Mel. & Auto. \\
\hline Heteropterys byrsonimifolia A.Juss. & murici-macho & NR & Mel. & Anec. \\
\hline Ceiba pubiflora (A. St.-Hil.) K. Schum. & barriguda & NR & Ornito. & Anec. \\
\hline Dombeya wallichii (Lindl.) K.Schum. & astrapeia & $\mathrm{E}$ & Mel. & Anec. \\
\hline $\begin{array}{l}\text { Eriotheca pubescens (Mart. \& Zucc.) Schott } \\
\text { \& Endl. }\end{array}$ & $\begin{array}{l}\text { paineira-do- } \\
\text { cerrado }\end{array}$ & NR & Mel. & Anec. \\
\hline Guazuma ulmifolia Lam. & mutamba & NR & Mel. & Auto. \\
\hline Luehea divaricata Mart. \& Zucc. & açoita-cavalo & NR & Mel. & Auto. \\
\hline Sterculia striata A.St.-Hil. \& Naudin & chichá & NR & Ent. & Zoo. \\
\hline Azadirachta indica A. Juss. & nim-indiano & $\mathrm{E}$ & Ent. & Zoo. \\
\hline Cedrela fissilis Vell. & cedro & NR & Mel. & Anec. \\
\hline Khaya sp. & mogno-africano & $\mathrm{E}$ & Mel. & Anec. \\
\hline Melia azedarach L. & cinamomo & $E$ & Ent. & Zoo. \\
\hline Artocarpus altilis (Parkinson) Fosberg & fruta-pão & $E$ & Anemo. & Zoo. \\
\hline Brosimum gaudichaudii Trécul & mama-cadela & NR & Anemo. & Zoo. \\
\hline Ficus benjamina $\mathrm{L}$ & ficus-benjamina & $\mathrm{E}$ & Mel. & Zoo. \\
\hline Ficus microcarpa L.f. & figueira-asiática & $\mathrm{E}$ & Mel. & Zoo. \\
\hline
\end{tabular}




\begin{tabular}{|c|c|c|c|c|c|}
\hline & Morus nigra L. & amoreira & $\mathrm{E}$ & Anemo. & Zoo. \\
\hline Moringaceae & Moringa oleifera Lam. & moringa & $\mathrm{E}$ & Mel. & Anec. \\
\hline \multirow{7}{*}{ Myrtaceae } & Eucalyptus sp. & eucalipto & $E$ & Mel. & Anec. \\
\hline & Eugenia dysenterica DC. & cagaita & NR & Mel. & Zoo. \\
\hline & Eugenia uniflora L. & pitangueira & $\mathrm{NO}$ & Mel. & Zoo. \\
\hline & Psidium guajava L. & goiabeira & $E$ & Mel. & Zoo. \\
\hline & Psidium cf. guineense Sw. & araçazeiro & NR & Mel. & Zoo. \\
\hline & Syzygium cumini (L.) Skeels & jamelão & $\mathrm{E}$ & Mel. & Zoo. \\
\hline & Syzygium jambos (L.) Alston & jambo & $E$ & Mel. & Zoo. \\
\hline Oleaceae & Ligustrum lucidum W.T.Aiton & ligustro & $E$ & Mel. & Zoo. \\
\hline Polygonaceae & Triplaris gardneriana Wedd. & pau-formiga & NR & Ent. & Anec. \\
\hline Proteaceae & Grevillea banksii R. Br. & grevílea & $E$ & Ornito. & Auto. \\
\hline \multirow[b]{2}{*}{ Rubiaceae } & Genipa americana L. & jenipapeiro & $\mathrm{NR}$ & Fal. & Zoo. \\
\hline & $\begin{array}{l}\text { Tocoyena formosa (Cham. \& Schltdl.) } \\
\text { K.Schum. }\end{array}$ & marmelo & NR & Fal. & Zoo. \\
\hline \multirow[b]{2}{*}{ Rutaceae } & Citrus limon (L.) Osbeck & limoeiro & $E$ & Mel. & Zoo. \\
\hline & Zanthoxylum riedelianum Engl. & $\begin{array}{l}\text { mamica-de- } \\
\text { porca }\end{array}$ & NR & Ent. & Zoo. \\
\hline \multirow{5}{*}{ Sapindaceae } & Filicium decipiens (Wight \& Arn.) Thwaites & $\begin{array}{c}\text { árvore- } \\
\text { samambaia }\end{array}$ & $E$ & Ent. & Zoo. \\
\hline & Koelreuteria bipinnata Franch. & árvore-da-china & $E$ & Ent. & Anec. \\
\hline & Magonia pubescens A.St.-Hil. & tingui & NR & Mel. & Anec. \\
\hline & Sapindus saponaria L. & saboneteiro & NR & Ent. & Zoo. \\
\hline & Talisia esculenta (Cambess.) Radlk. & pitombeira & NR & Ent. & Zoo. \\
\hline Solanaceae & Solanum lycocarpum A.St.-Hil. & lobeira & NR & Mel. & Zoo. \\
\hline Vochysiaceae & Qualea multiflora Mart. & pau-terra & NR & Mel. & Anec. \\
\hline
\end{tabular}

Nota: Nativa da região (NR); Nativa de outras regiões do Brasil (NO); Exótica (E); Síndromes de Polinização (SP); Anemofilia (Anemo.); Cantarofilia (Can.); Entomofilia (Ent.); Falenofilia (Fal.); Melitofilia (Mel.); Ornitofilia (Ornito.); Quiropterofilia (Quirop.); Síndromes de Dispersão (SD); Anemocoria (Anec.); Autocoria (Auto.); Barocoria (Baro.); Hidrocoria (Hidro.); Zoocoria (Zoo.).

Em relação à origem, as espécies que compõem a arborização do Campus Regional, são predominantemente nativas do Brasil, correspondendo a $57,4 \%$ do total. A preferência por espécies autóctones e a grande riqueza florística também foi reportada em levantamento no Campus Sede da UFMG em Belo Horizonte (LOMBARDI; MORAIS, 2003), porém, fato contrário foi observado na arborização urbana de cidades do norte de Minas Gerais, como Jequitaí (SANTOS; FONSECA; GONÇALVES, 2019) e Montes Claros (VELOSO et al., 2015; FIRMO et al., 2019), onde predominaram táxons exóticos e menor riqueza de espécies. A maior proporção de espécies nativas nos campi da UFMG pode estar relacionada à proximidade com fragmentos florestais que atuam como fonte de propágulos (CUPERTINO; EISENLOHR, 2013), distinguindo das demais áreas amostradas na região, que estão imersas em meio antrópico.

Dentre as espécies nativas do Brasil, cerca de $87 \%$ ocorrem naturalmente no norte de Minas Gerais, fator que associado ao aspecto ecotonal da região, fazem do local um pequeno mostruário da riqueza florística existente nesta porção do estado. Nas áreas verdes do campus é possível encontrar espécies típicas do domínio da Caatinga, como Spondias tuberosa 
(umbuzeiro) e Amburana cearensis (amburana-de-cheiro); do Cerrado, como Caryocar brasiliense (pequizeiro) e uma grande diversidade de espécies dos gêneros Handroanthus e Tabebuia (ipês); além de representantes do domínio da Mata Atlântica, como Cedrela fissilis (cedro-rosa) e Calophyllum brasiliense (guanandi) (Figura 2).

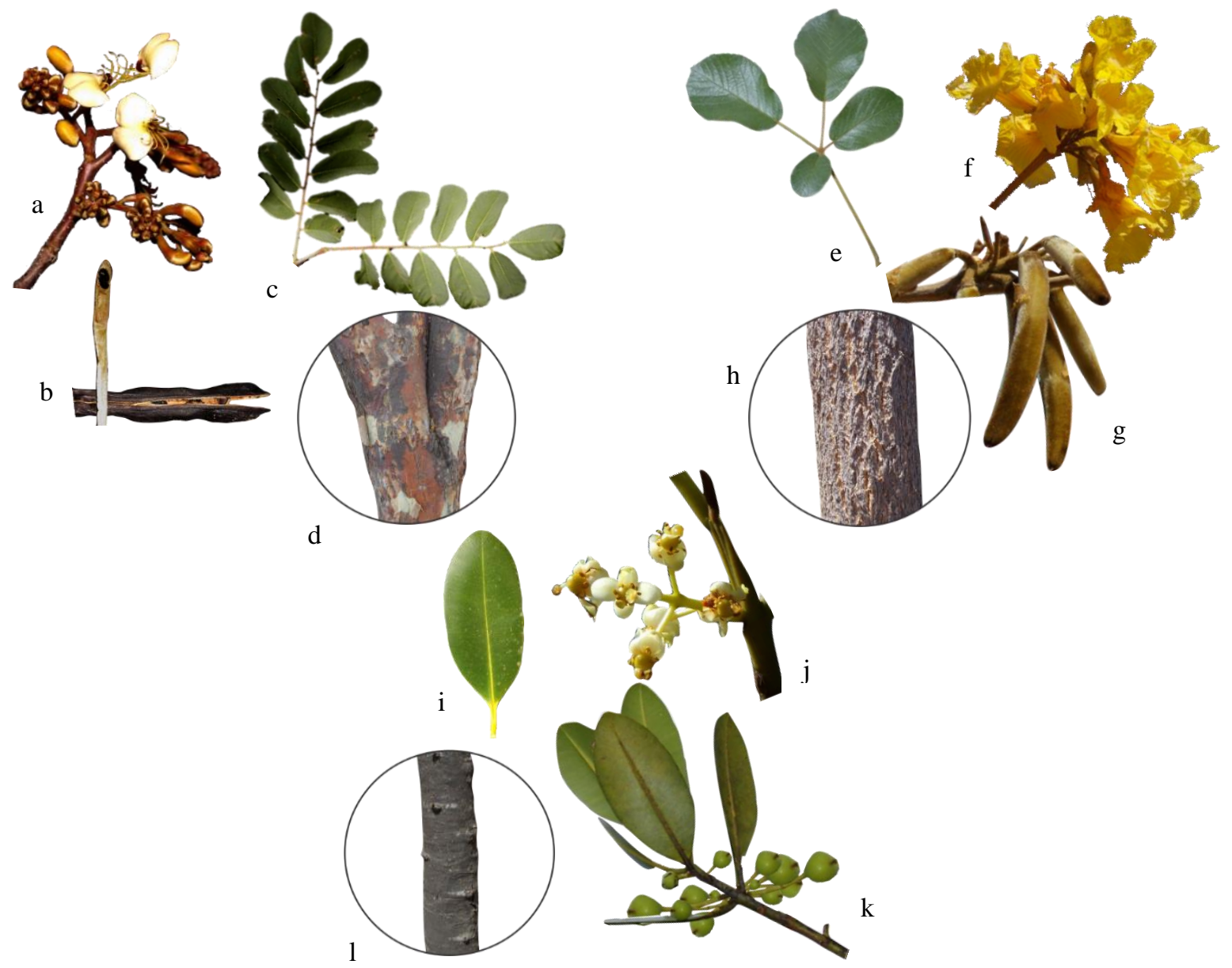

Figura 2. Espécies típicas dos domínios morfoclimáticos que ocorrem no Campus Regional da Universidade Federal de Minas Gerais, em Montes Claros, MG. Caatinga: Amburana cearensis (a, b, c, d); Cerrado: Handroanthus ochraceus (e, f, g, h); Mata Atlântica: Calophyllum brasiliense $(\mathrm{i}, \mathrm{j}, \mathrm{k}, \mathrm{l})$

Figure 2. Typical species of the morphoclimatic domains that occur at the Regional Campus of the Federal University of Minas Gerais, in Montes Claros, MG. Caatinga: Amburana cearensis (a, b, c, d); Cerrado: Handroanthus ochraceus (e, $\mathrm{f}, \mathrm{g}, \mathrm{h}$ ); Atlantic Forest: Calophyllum brasiliense (i, j, k, l)

Por se tratar de um ambiente de disseminação do conhecimento científico, a composição florística observada constitui ferramenta para ações de educação ambiental sobre a riqueza e complexidade ecológica do norte de Minas Gerais. A preferência pelo emprego de espécies autóctones no paisagismo de campi universitários contribui para a valoração da flora regional e o conhecimento das funções ecológicas que elas desempenham (CASTRO; MORO; ROCHA, 2011). 
No Campus Regional foram inventariadas espécies com diferentes graus de ameaça de extinção. Segundo o CNCFlora (2020), listadas como Quase Ameaçada (NT) estão Handroanthus impetiginosus (ipê-roxo) e Amburana cearensis (amburana-de-cheiro); como Vulnerável (VU) Cedrela fissilis (cedro-rosa) e Butia capitata (coquinho-azedo). Além destas, a unidade acadêmica possui espécies que são protegidas por leis e decretos estaduais e federais, como Caryocar brasiliense (pequizeiro), Astronium urundeuva (aroeira-do-sertão), Astronium fraxinifolium (gonçalo-alves) e as dos gêneros Handroanthus e Tabebuia conhecidas popularmente como ipês-amarelos (sensu HENRIQUES; VICHIATO; VICHIATO, 2018).

A presença de espécies ameaçadas de extinção na arborização do Campus Regional da UFMG revela o papel conservacionista de seus espaços verdes, contribuindo para a manutenção in situ e ex situ da flora e, consequentemente, da fauna associada (CASTRO; MORO; ROCHA, 2011; CUPERTINO; EISENLOHR, 2013).

O uso de espécies exóticas na arborização urbana é recomendado com cautela, pois muitas possuem o potencial invasor, além de serem mais susceptíveis ao ataque de agentes patogênicos (PATRÍCIO, 2017). Porém, a introdução planejada de espécies alóctones de forma complementar às nativas em ambientes antropizados apresentam também diversas vantagens (SJÖMAN et al., 2016). Entre os benefícios estão o fornecimento de recursos alimentares para a fauna e a conservação da biodiversidade, fatores observados no Campus Regional, onde parte das espécies exóticas são frutíferas, como Mangifera indica (mangueira), Spondias dulcis (cajámanga) e Syzygium jambos (jambo). Além disso, táxons exóticos ameaçados de extinção (IUCN 2020), também são cultivados nas vias e jardins da unidade acadêmica, como Khaya sp. (mognoafricano), Dypsis lutescens (palmeira-areca) e Dypsis decaryi (palmeira-triângulo).

Em relação à interação das espécies com os polinizadores, a melitofilia foi a síndrome de polinização predominante nas espécies nativas e exóticas (Figura 3). As abelhas são polinizadores que se comportam de forma mais generalista e visitam espécies com variadas morfologias florais, o que as tornam os principais agentes da polinização em ambientes tropicais, naturais ou antropizados (GOTTSBERGER; SILBERBAUER-GOTTSBERGER, 2018; SANTOS; FONSECA; GONÇALVES, 2019).

Entomofilia foi a segunda síndrome mais representativa, com 22,3\% das espécies amostradas. Táxons entomófilos, isto é, polinizados por diversos pequenos insetos, encontramse entre os mais recorrentes em espaços verdes urbanos (MONALISA-FRANCISCO et al., 2019) e fragmentos florestais nativos da Caatinga, Cerrado e Mata Atlântica (KINOSHITA et al., 2006; GOTTSBERGER; SILBERBAUER-GOTTSBERGER, 2018; LEAL et al., 2018). 

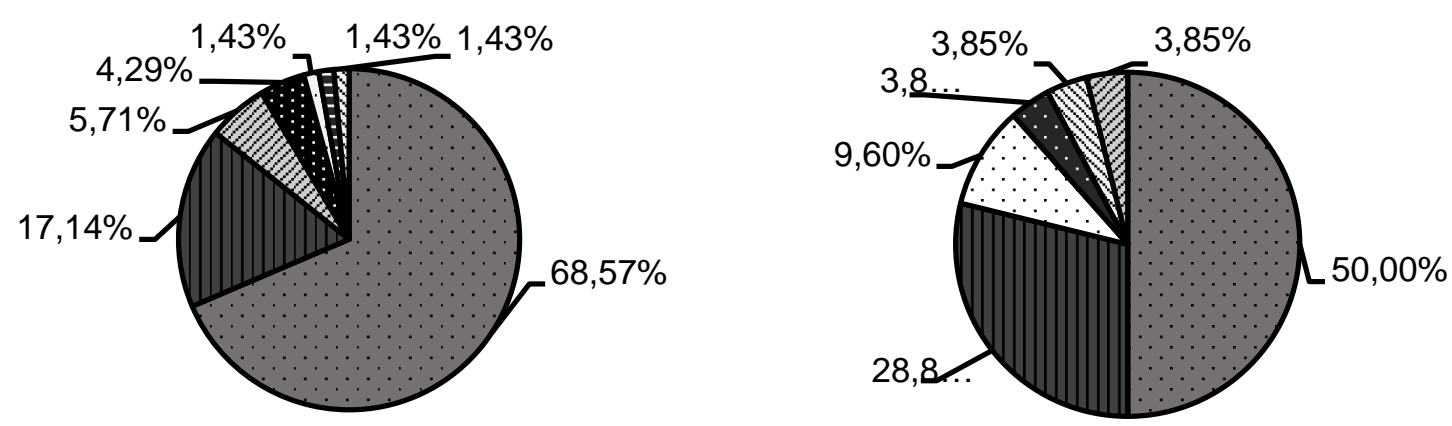

\section{Melitofilia \\ Quiropterofilia : \\ Falenofil: \\ Anemofilia}

\section{$\$$ Ornitofilia $\equiv \quad$ Cantarofilia}

Figura 3. Síndromes de polinização das espécies nativas (à esquerda) e exóticas (à direita) da arborização do Campus Regional da Universidade Federal de Minas Gerais, Montes Claros, MG

Figure 3. Pollination syndromes of native (left) and exotic (right) species from the afforestation at the Regional Campus of the Federal University of Minas Gerais, Montes Claros, MG

Anemofilia e quiropterofilia representaram cada uma 4,9\% do levantamento. As espécies anemófilas levantadas foram praticamente todas de origem exótica, com exceção de Brosimum gaudichaudii, corroborando outros trabalhos, como o de Santos, Fonseca e Gonçalves (2019), que demonstraram a raridade desta síndrome em ambientes urbanos de regiões tropicais. Já as quiropterófilas foram representadas em sua maioria por espécies nativas $(66,7 \%)$, confirmando a afirmação de Leal et al. (2018), de que os morcegos são importantes polinizadores, especialmente na Caatinga. Falenofilia $(4,1 \%)$, ornitofilia $(2,5 \%)$ e cantarofilia $(0,8 \%)$ foram as síndromes mais raras, o que pode ser explicado pela baixa representatividade de espécies arbóreas com estruturas e recursos florais atrativos para mariposas, pássaros e besouros, respectivamente.

Em relação às síndromes de dispersão, a zoocoria predominou, perfazendo 45,9\% das espécies. Ao analisar separadamente as espécies por origem, verifica-se que a representatividade das síndromes é distinta (Figura 4).

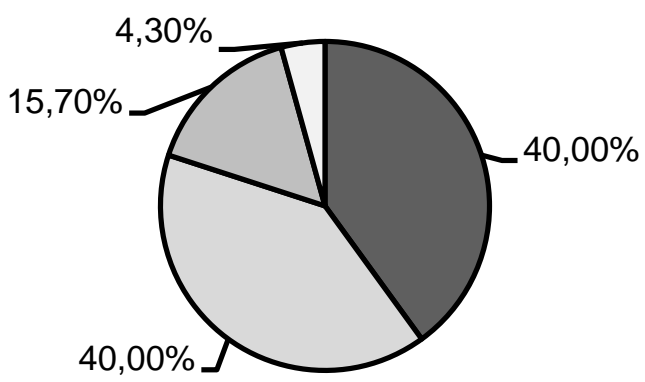

\section{Zoocoria}

Anemocoria

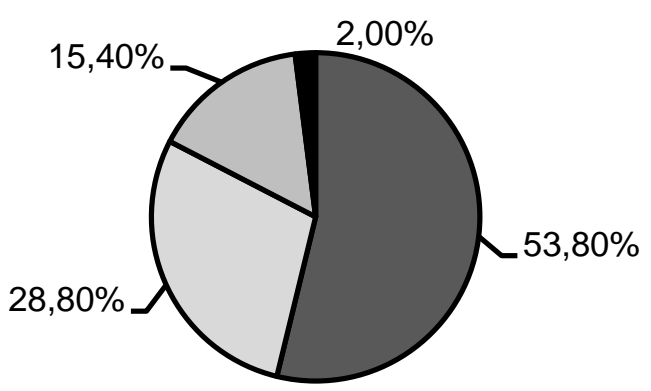

Autocoria $\square$ Barocoria
Hidrocoria

Figura 4. Síndromes de dispersão das espécies nativas (à esquerda) e exóticas (à direita) da arborização do Campus Regional da Universidade Federal de Minas Gerais, Montes Claros, MG

Figure 4. Dispersal syndromes of native (left) and exotic (right) species from the afforestation at the Regional Campus of the Federal University of Minas Gerais, Montes Claros, MG 
Entre as espécies exóticas, a zoocoria foi também a mais reportada, sendo a família Arecaceae responsável por mais de um terço das espécies $(32,1 \%)$. O fato das palmeiras alóctones possuírem frutos carnosos e serem frequentemente usadas na arborização urbana justifica a sua considerável participação na amostragem. Já a anemocoria e a autocoria estiveram representadas em grande parte pelas famílias Bignoniaceae $(26,7 \%)$ e Fabaceae (75\%), respectivamente, grupos tipicamente predominantes com estas síndromes em ambientes urbanos, conforme evidenciado por Monalisa-Francisco et al. (2019). Por fim, a hidrocoria foi a síndrome menos representativa, com apenas uma espécie em todo levantamento, Cocus nucifera (coqueiro).

Dentre as espécies nativas, observou-se que a anemocoria e a zoocoria estiveram igualmente representadas, com 40\% cada. Espécies zoocóricas figuram entre as mais frequentes em fragmentos nativos de cerrado sensu stricto e florestas do domínio atlântico (KINOSHITA et al., 2006; GOTTSBERGER; SILBERBAUER-GOTTSBERGER, 2018). Já espécies anemocóricas são típicas de formações adaptadas ao clima mais seco, como as presentes nos domínios da Caatinga e do Cerrado, condição ambiental que favorece este modo de dispersão (HOWE; SMALLWOOD, 1982). Essa condição intermediária pode estar relacionada ao caráter ecotonal observado na flora da área de estudo.

Autocoria e barocoria foram as síndromes de dispersão menos frequentes. Espécies autocóricas são pouco reportadas na flora lenhosa do Cerrado e das florestas tropicais úmidas (GOTTSBERGER; SILBERBAUER-GOTTSBERGER, 2018), mas são consideravelmente expressivas, assim como as barocóricas, na Caatinga (LEAL et al., 2018).

A análise das síndromes demonstrou o potencial de interação e manutenção da fauna pela comunidade arbórea, tanto dos polinizadores quanto de dispersores. Além de garantir a frutificação, essa fauna promove a dispersão de sementes e a colonização vegetal das áreas degradadas da unidade acadêmica e adjacências. Ao se planejar os ecossistemas urbanos devese levar em conta as interações ecológicas, a fim de garantir a manutenção da biodiversidade, favorecendo também a atuação dos ambientes urbanos como corredores ecológicos.

\section{CONCLUSÕES}

O Campus Regional da UFMG, em Montes Claros, abriga em suas principais vias e jardins 122 espécies arbóreas e arborescentes, distribuídas em 33 famílias botânicas. Quanto à origem, $57,4 \%$ são nativas do Brasil e $42,6 \%$ são exóticas ou naturalizadas. A presença de espécies nativas dos domínios da Caatinga, Cerrado e Mata Atlântica na arborização da unidade 
acadêmica reforça a natureza ecotonal própria da região norte de Minas Gerais, constituindo uma amostra demonstrativa e didática da riqueza florística regional.

A presença de exemplares de espécies nativas e exóticas ameaçadas de extinção em seus ambientes naturais de origem, além de outras protegidas por leis e decretos estaduais e federais, evidenciam que essa comunidade arbórea cumpre o papel conservacionista proposto pela silvicultura urbana.

Quanto às síndromes de polinização e dispersão, melitofilia e zoocoria foram as mais representativas, respectivamente, demostrando a importância dessa flora arbórea para a manutenção da fauna local.

\section{AGRADECIMENTOS}

À UFMG pelo apoio estrutural, ao Grupo de Estudos em Reprodução, Fenologia e Florística de Vegetações Sazonais (REFFLOR) pelo apoio técnico e à Junimaura Resende da Silva e Eduardo José Micelli Munhoz pelo apoio no levantamento de campo.

\section{REFERÊNCIAS}

ARRUDA, D. M.; FERREIRA-JUNIOR, W. G.; DUQUE-BRASIL, R.; SCHAEFER, C. E. R. Phytogeographical patterns of dry forests sensu stricto in northern Minas Gerais State, Brazil. Anais da Academia Brasileira de Ciências, Rio de Janeiro, v. 85, n. 2, p. 623-634, 2013.

CASTRO, A. S. F.; MORO, M. F.; ROCHA, F. C. L. Plantas dos espaços livres da Reitoria da Universidade de Fortaleza (UNIFOR), Ceará, Brasil. Revista Brasileira de Biociências, Porto Alegre, v. 9, n. 1, p. 126-129, 2011.

CENTRO NACIONAL DE CONSERVAÇÃO DA FLORA (CNCFLORA). Lista Vermelha. Disponível em: <http://cncflora.jbrj.gov.br/portal/>. Acesso em 20 mar. 2020.

CUPERTINO, M. A.; EISENLOHR, P. V. Análise florística comparativa da arborização urbana nos campi universitários do Brasil. Bioscience Journal, Uberlândia, v. 29, p. 739-750, 2013.

DUQUE-BRASIL, R.; SOLDATI, G. T.; ESPÍRITO-SANTO, M. M.; REZENDE, M. Q.; D'ÂNGELONETO, S.; COELHO, F. M. G. Composição, uso e conservação de espécies arbóreas em quintais de agricultores familiares na região da mata seca norte-mineira, Brasil. Sitientibus Série Ciências Biológicas, Feira de Santana, v. 11, n. 2, p. 287-297, 2012.

DURÃES, M. C. O.; SALES, N. L.; NETO, S. D.; FIGUEIREDO, M. A. P.; DURÃES, M. C. O.; SALES, N. de L. P.; D'ÂNGELO NETO, S.; FIGUEIREDO, M. A. P. Levantamento florístico do estrato arbóreo de três fragmentos de floresta ciliar como subsídio à recomposição da vegetação do Rio Cedro, Montes Claros - MG. Ciência Florestal, Santa Maria, v. 24, n. 1, p. 47-58, 2014. 
FAEGRI, K.; PIJL, L. The principles of pollination ecology. Oxford, Pergamon Press. 1976.

FIRMO, D. H. T.; FREITAS, D. A.; DURÃES, A. F. S.; SILVA, A. C.; ALMEIDA, E. F. A. Arborização urbana: uma imprescindível prática de manejo dos espaços urbanos. Brazilian Journal of Animal and Environmental Research, São José dos Pinhais, v. 2, n. 5, p. 15841601, 2019.

FLORA DO BRASIL. FLORA DO BRASIL 2020 em construção. Jardim Botânico do Rio de Janeiro. Disponível em: <http://floradobrasil.jbrj.gov.br/>. Acesso em 10 mar. 2020.

GILL, S.; HANDLEY, J. F.; ENNOS, A. R.; PAULEIT, S. Adapting Cities for Climate Change: The Role of the Green Infrastructure. Built Environment, Abingdon, v. 33, n. 1, p. 115-133, 2007.

GOTTSBERGER, G.; SILBERBAUER-GOTTSBERGER, I.; GOTTSBERGER, G.; SILBERBAUER-GOTTSBERGER, I. How are pollination and seed dispersal modes in Cerrado related to stratification? Trends in a cerrado sensu stricto woodland in southeastern Brazil, and a comparison with Neotropical forests. Acta Botanica Brasilica, Brasília, v. 32, n. 3, p. 434-445, 2018.

HENRIQUES, L. C. M.; VICHIATO M. R. M.; VICHIATO, M. Conservação de espécies florestais protegidas ou ameaçadas de extinção em Belo Horizonte, MG. Tecnologia \& Ciência Agropecuária, João Pessoa, v. 12, n. 2, p. 15-24, 2018.

HOWE, H., SMALLWOOD, J. Ecology of seed dispersal. Annual Review of Ecology and Systematics, Palo Alto, n.13, p. 201-228, 1982.

INTERNATIONAL UNION FOR CONSERVATION OF NATURE'S (IUCN). The IUCN Red List of Threatened Species. Version 2019-3. Disponível em: <https://www.iucnredlist.org>. Acesso em 20 mar. 2020.

KINOSHITA, L. S.; TORRES, R. B.; FORNI-MARTINS, E. R.; SPINELLI, T.; AHN, Y. J.; CONSTÂNCIO, S. S. Composição florística e síndromes de polinização e de dispersão da mata do Sítio São Francisco, Campinas, SP, Brasil. Acta Botanica Brasilica, Brasília, v. 20, n. 2, p. 313-327, 2006.

LEAL, I. R.; LOPES, A. V.; MACHADO, I. C.; TABARELLI, M. Interações planta-animal na Caatinga: visão geral e perspectivas futuras. Ciência e Cultura, Campinas, v. 70, n. 4, p. 35-40, 2018.

LOMBARDI, J. A.; MORAIS, P. O. Levantamento florístico das plantas empregadas na arborização do campus da Universidade Federal de Minas Gerais, Belo Horizonte-MG. Lundiana, Belo Horizonte, v. 4, n. 2, p. 83-88, 2003.

MONALISA-FRANCISCO, N.; RAMOS, F. N. Composition and Functional Diversity of the Urban Flora of Alfenas-MG, Brazil. Floresta e Ambiente, Seropédica, v. 26, n. 3, p. 1-11, 2019.

PATRÍCIO, P. P. M. Florística e diagnóstico da arborização da Universidade Federal de Mato Grosso, campus Cuiabá. 2017. 106 f. Dissertação (Mestrado em Ciências Florestais e Ambientais) - Universidade Federal de Mato Grosso, Cuiabá, 2017.

PREFEITURA MUNICIPAL DE MONTES CLAROS (PMMC). Conheça a Cidade. Disponível em: $<$ https://portal.montesclaros.mg.gov.br/conheca-a-cidade>. Acesso em 28 mar. 2020.

SANTOS, G. R. dos; FONSECA, R. S.; GONÇALVES, C. B. Arborização urbana em Jequitaí - 
MG: atributos funcionais e diversidade. Revista da Sociedade Brasileira de Arborização Urbana, Curitiba, v. 14, n. 1, p. 1-13, 2019.

SJÖMAN, H.; MORGENROTH, J.; SJÖMAN, J. D.; S/EBØ, A.; KOWARIK, I. Diversification of the urban forest-Can we afford to exclude exotic tree species? Urban Forestry and Urban Greening, Amsterdã, v. 18, n. July, p. 237-241, 2016.

THE PLANT LIST. Search. Disponível em: http://www.theplantlist.org/. Acesso em 25 mar. 2020.

VAN DER PIJL, L. Principles of dispersal in higher plants. Berlim: Springer-Verlag, 1982. $215 p$.

VELOSO, M. das D. M.; BRAGA, L. D. L.; RODRIGUES, P. M. S.; SANTOS, M. R.; MIRANDA, W. O.; BRANDÃO, D. O.; NUNES, Y. R. F. Caracterização da arborização urbana em três ambientes na cidade de Montes Claros, MG. Revista da Sociedade Brasileira de Arborização Urbana, Piracicaba, v. 9, n. 2, p. 118-133, 2015. 